\title{
Structure of prescribed gradient domains for non-integrable vector fields
}

\section{Silvano Delladio ${ }^{1}$}

Received: 13 November 2015 / Accepted: 22 February 2017 / Published online: 1 October 2018 (c) Fondazione Annali di Matematica Pura ed Applicata and Springer-Verlag GmbH Germany, part of Springer Nature 2018

\section{Abstract}

Let $F \in C^{1}\left(\Omega, \mathbb{R}^{n}\right)$ and $f \in C^{2}(\Omega)$, where $\Omega$ is an open subset of $\mathbb{R}^{n}$ with $n$ even. We describe the structure of the set of points in $\Omega$ at which the equality $D f=F$ and a certain non-integrability condition on $F$ hold. This result generalizes the second statement of Balogh (J Reine Angew Math 564:63-83, 2003, Theorem 3.1).

Keywords Prescribed gradient domains - Characteristic set · Real analysis on submanifolds Mathematics Subject Classification Primary 28A75 · 15A69 $\cdot 47 \mathrm{H} 60$

\section{Introduction}

Let us consider an open subset $\Omega$ of $\mathbb{R}^{n}$ and a vector field $F \in C^{1}\left(\Omega, \mathbb{R}^{n}\right)$. Then, whatever the choice of $f \in C^{2}(\Omega)$, the set

$$
A_{f, F}:=\{x \in \Omega \mid D f(x)=F(x)\}
$$

has to be somehow "scarcely dense" at each point $x \in \Omega$ satisfying condition

$$
(D F(x))^{t} \neq D F(x) \text {. }
$$

Actually, if such a condition occurs then the point $x$ cannot be in the interior of $A_{f, F}$, by the Schwarz theorem on mixed derivatives. More interestingly, by [3, Theorem 2.1], the point $x$ cannot be a $(n+1)$-density point of the set $A_{f, F}$, i.e., it must be

$$
\limsup _{r \rightarrow 0+} \frac{\mathcal{L}^{n}\left(B(x, r) \backslash A_{f, F}\right)}{r^{n+1}}>0 .
$$

In the special case when $n=2 m$ and

$$
F_{0}\left(x_{1}, \ldots, x_{2 m}\right)=\left(2 x_{m+1}, \ldots, 2 x_{2 m},-2 x_{1}, \ldots,-2 x_{m}\right)
$$

$\bowtie \quad$ Silvano Delladio

silvano.delladio@unitn.it

1 Dipartimento di Matematica, Università degli Studi di Trento, Povo, Trento, Italy 
condition (1.1) is trivially satisfied everywhere by $F_{0}$ and, according to the Balogh's result [1, Theorem 3.1], the set $A_{f, F_{0}}$ is covered by countably many $m$-dimensional Lipschitz graphs. In particular, the Hausdorff dimension of $A_{f, F_{0}}$ cannot exceed $m$. This theorem implies immediately that, given a $C^{2}$ hypersurface $S$ in $\mathbb{R}^{2 m+1}$, the set of points at which the tangent space of $S$ coincides with the space spanned by the left-invariant horizontal vector fields of the Heisenberg group $\mathbb{H}^{m}$ over $\mathbb{R}^{2 m+1}$ (namely the characteristic set of $S$ ) has Hausdorff dimension less or equal to $m$, compare [1, Theorem 1.2].

The main goal of this short paper is a theorem generalizing Balogh's result above and describing the structure of the set of points in $A_{f, F}$ satisfying a certain condition which is stronger than (1.1). In order to formulate such a condition and to state our result, let us consider the canonical basis $\left\{e_{1}, \ldots, e_{n}\right\}$ of $\mathbb{R}^{n}$ and a real $n \times n$ matrix $M$. Then define

$$
\Gamma(M):=\sum_{i=1}^{n} M e_{i} \wedge e_{i}
$$

and (if $h$ is a positive integer)

$$
\Gamma(M)^{h}:=\underbrace{\Gamma(M) \wedge \cdots \wedge \Gamma(M)}_{h \text { times }} .
$$

Now we can finally state our structure theorem. It establishes that if $n=2 m$, then the set $A_{f, F}^{*}$ of all points $x \in A_{f, F}$ such that

$$
\Gamma(D F(x))^{m} \neq 0
$$

is covered by a finite family of m-dimensional regularly imbedded $C^{1}$ submanifolds of $\mathbb{R}^{2 m}$ (Theorem 4.1 below).

In the special case considered by Balogh, i.e., when $F=F_{0}$, the condition (1.2) is verified at every $x \in \mathbb{R}^{n}$. Indeed a standard computation yields

$$
\Gamma\left(D F_{0}(x)\right)^{m}=C(m) e_{1} \wedge \cdots \wedge e_{2 m}
$$

for all $x \in \mathbb{R}^{n}$, where $C(m)$ is a positive constant depending only on $m$. Hence $A_{f, F_{0}}=A_{f, F_{0}}^{*}$, thus Balogh's result follows trivially from our theorem (Corollary 4.1 below).

The proof of our structure theorem is provided in Sect. 4 and is an elementary argument combining the classical implicit function theorem with some basic results from multilinear algebra which are developed in Sect. 3.

We have eventually to mention a few main results strictly related to this subject that have been published after [1]. In paper [4], Franchi et al. extended the Balogh's covering type argument to all stratified groups of step two. The generalization to arbitrary stratified groups has been proved by V. Magnani through a different approach based on a coarea inequality [7]. More recently, an estimate for the size of tangencies of submanifolds with respect to a noninvolutive distribution, which generalizes the Balogh's theorem above, has been provided in [2].

\section{Notation}

If $m, n$ are positive integers with $m \leq n$ then $I(n, m)$ is the set of integer multi-indices $\left(\alpha_{1}, \ldots, \alpha_{m}\right)$ such that $1 \leq \alpha_{1}<\ldots<\alpha_{m} \leq n$. If $\alpha \in I(n, m)$ then $\bar{\alpha}$ is the complement in $I(n, n-m)$ of $\alpha$. Moreover $\sigma(\alpha, \bar{\alpha})$ is the sign of the permutation of $(1, \ldots, n)$ into $(\alpha, \bar{\alpha})$. The space of $m$-vectors in $\mathbb{R}^{n}$ is denoted by $\Lambda^{m} \mathbb{R}^{n}$. If $\left\{e_{1}, \ldots, e_{n}\right\}$ is a basis of $\mathbb{R}^{n}$ and 
$\alpha=\left(\alpha_{1}, \ldots, \alpha_{m}\right) \in I(n, m)$, then we put $e_{\alpha}:=e_{\alpha_{1}} \wedge \cdots \wedge e_{\alpha_{m}}$. Recall that $\left\{e_{\alpha}\right\}_{\alpha \in I(n, m)}$ is a basis of $\Lambda^{m} \mathbb{R}^{n}$. Let $M_{n}(\mathbb{R})$ denote the space of all $n \times n$ matrices with real entries and consider $M \in M_{n}(\mathbb{R})$. Then $M^{t}$ is the transpose of $M$. Moreover, if $1 \leq m \leq n$, define the linear operator $\Lambda^{m} M: \Lambda^{m} \mathbb{R}^{n} \rightarrow \Lambda^{m} \mathbb{R}^{n}$ as the one such that $\Lambda^{m} M\left(e_{\alpha}\right)=M e_{\alpha_{1}} \wedge \cdots \wedge M e_{\alpha_{m}}$, for all $\alpha=\left(\alpha_{1}, \ldots, \alpha_{m}\right) \in I(n, m)$. Observe that, for all $\beta \in I(n, m)$, one has

$$
\Lambda^{m} M\left(e_{\beta}\right)=\sum_{\alpha \in I(n, m)}\left(\operatorname{det} M_{\alpha, \beta}\right) e_{\alpha}
$$

where $M_{\alpha, \beta}$ is the $m \times m$ submatrix of $M$ with rows $\alpha_{1}, \ldots, \alpha_{m}$ and columns $\beta_{1}, \ldots, \beta_{m}$. The symmetric group of degree $m$ is denoted by $S_{m}$. Finally, if $\Omega$ is an open subset of $\mathbb{R}^{n}$, $x \in \Omega$ and $\psi \in C^{1}\left(\Omega, \mathbb{R}^{m}\right)$, then $D \psi(x)$ denotes the matrix of the differential of $\psi$ at $x$ (with respect to the canonical basis).

\section{Some basic preliminaries from multilinear algebra}

Proposition 3.1 Let $\left\{e_{1}, \ldots, e_{n}\right\}$ be the canonical basis of $\mathbb{R}^{n}$. Consider the operator $\Gamma$ : $M_{n}(\mathbb{R}) \rightarrow \Lambda^{2} \mathbb{R}^{n}$ defined as

$$
\Gamma(M):=\sum_{i=1}^{n} M e_{i} \wedge e_{i}, \quad M \in M_{n}(\mathbb{R}) .
$$

Then $\Gamma$ has the following properties:

(1) It is linear, namely if $r, s \in \mathbb{R}$ and $M, N \in M_{n}(\mathbb{R})$ then

$$
\Gamma(r M+s N)=r \Gamma(M)+s \Gamma(N) .
$$

(2) For all $M \in M_{n}(\mathbb{R})$ one has

$$
\Gamma(M)=\sum_{\substack{i, j=1 \\ i<j}}^{n}\left(M_{i j}-M_{j i}\right) e_{i} \wedge e_{j}
$$

where $M_{i j}:=\left(M e_{j}\right) \cdot e_{i}$. Hence:

(i) $\Gamma\left(M^{t}\right)=-\Gamma(M)$;

(ii) $\Gamma(M)=0$ if and only if $M$ is symmetric.

(3) If $\left\{u_{1}, \ldots, u_{n}\right\}$ is any arbitrary orthonormal basis in $\mathbb{R}^{n}$ and $M \in M_{n}(\mathbb{R})$, then

$$
\sum_{i=1}^{n} M u_{i} \wedge u_{i}=\Gamma(M) .
$$

Moreover, in the special case when $n$ is even, i.e., $n=2 m$, the following identities hold for all $M \in M_{2 m}(\mathbb{R})$ :

$$
\begin{aligned}
\Gamma(M)^{m}:=\underbrace{\Gamma(M) \wedge \cdots \wedge \Gamma(M)}_{\text {mtimes }} & =C(m) \sum_{\alpha \in I(2 m, m)}\left[\left(\Lambda^{m} M\right) e_{\alpha}\right] \wedge e_{\alpha} \\
& =C(m)\left(\sum_{\alpha \in I(2 m, m)} \sigma(\alpha, \bar{\alpha}) \operatorname{det} M_{\alpha, \bar{\alpha}}\right) e_{1} \wedge \cdots \wedge e_{2 m}
\end{aligned}
$$


with $C(m):=m !(-1)^{\frac{m(m-1)}{2}}$.

Proof Assertion (1) follows immediately from the definition of $\Gamma$, while (2) is a standard computation:

$$
\begin{aligned}
\Gamma(M) & =\sum_{i, j=1}^{n} M_{j i} e_{j} \wedge e_{i} \\
& =\sum_{\substack{i, j=1 \\
i<j}}^{n} M_{j i} e_{j} \wedge e_{i}+\sum_{\substack{i, j=1 \\
i>j}}^{n} M_{j i} e_{j} \wedge e_{i} \\
& =\sum_{\substack{i, j=1 \\
i<j}}^{n} M_{j i} e_{j} \wedge e_{i}+\sum_{\substack{i, j=1 \\
i<j}}^{n} M_{i j} e_{i} \wedge e_{j} \\
& =\sum_{\substack{i, j=1 \\
i<j}}^{n}\left(M_{i j}-M_{j i}\right) e_{i} \wedge e_{j} .
\end{aligned}
$$

Also (3) is very easy:

$$
\begin{aligned}
\sum_{i=1}^{n} M e_{i} \wedge e_{i} & =\sum_{i, h, k=1}^{n}\left(e_{i} \cdot u_{h}\right)\left(e_{i} \cdot u_{k}\right) M u_{h} \wedge u_{k} \\
& =\sum_{h, k=1}^{n}\left[\sum_{i=1}^{n}\left(e_{i} \cdot u_{h}\right)\left(e_{i} \cdot u_{k}\right)\right] M u_{h} \wedge u_{k} \\
& =\sum_{h, k=1}^{n}\left(u_{h} \cdot u_{k}\right) M u_{h} \wedge u_{k} \\
& =\sum_{h=1}^{n} M u_{h} \wedge u_{h} .
\end{aligned}
$$

Finally, one has

$$
\begin{aligned}
\Gamma(M)^{m} & =\left(\sum_{i_{1}=1}^{2 m} M e_{i_{1}} \wedge e_{i_{1}}\right) \wedge \cdots \wedge\left(\sum_{i_{m}=1}^{2 m} M e_{i_{m}} \wedge e_{i_{m}}\right) \\
& =(-1)^{1+2+\cdots+m-1} \sum_{i_{1}, \ldots, i_{m}=1}^{2 m} M e_{i_{1}} \wedge \cdots \wedge M e_{i_{m}} \wedge e_{i_{1}} \wedge \cdots \wedge e_{i_{m}} \\
& =(-1)^{\frac{m(m-1)}{2}} \sum_{\alpha \in I(2 m, m)} \sum_{\lambda \in S_{m}} M e_{\alpha_{\lambda(1)}} \wedge \cdots \wedge M e_{\alpha_{\lambda(m)}} \wedge e_{\alpha_{\lambda(1)}} \wedge \cdots \wedge e_{\alpha_{\lambda(m)}} \\
& =(-1)^{\frac{m(m-1)}{2}} \sum_{\alpha \in I(2 m, m)} \#\left(S_{m}\right)\left[\left(\Lambda^{m} M\right) e_{\alpha}\right] \wedge e_{\alpha} \\
& =(-1)^{\frac{m(m-1)}{2}} m ! \sum_{\alpha \in I(2 m, m)}\left[\left(\Lambda^{m} M\right) e_{\alpha}\right] \wedge e_{\alpha}
\end{aligned}
$$


and

$$
\begin{aligned}
{\left[\left(\Lambda^{m} M\right) e_{\alpha}\right] \wedge e_{\alpha} } & =\sum_{\beta \in I(2 m, m)}\left(\operatorname{det} M_{\beta, \alpha}\right) e_{\beta} \wedge e_{\alpha}=\left(\operatorname{det} M_{\bar{\alpha}, \alpha}\right) e_{\bar{\alpha}} \wedge e_{\alpha} \\
& =\sigma(\bar{\alpha}, \alpha)\left(\operatorname{det} M_{\bar{\alpha}, \alpha}\right) e_{1} \wedge \cdots \wedge e_{2 m}
\end{aligned}
$$

Remark 3.1 From (1) and (2) of Proposition 3.1, it follows at once the following identity which will be useful below:

$$
\Gamma\left(M-M^{t}\right)=2 \Gamma(M)=-2 \Gamma\left(M^{t}\right)
$$

for all $M \in M_{n}(\mathbb{R})$.

Remark 3.2 Let $h \geq 2$ and $n \geq 2 h$. Then the identity $\Gamma(M)^{h}=0$ holds whenever $M$ is symmetric (by (2) of Proposition 3.1), but it can occur even when $M$ is nonsymmetric. For example, for

$$
M_{i j}= \begin{cases}1 & \text { if } i=1 \text { and } j=2 \\ 0 & \text { otherwise }\end{cases}
$$

one has $\Gamma(M)=e_{1} \wedge e_{2}$, hence $\Gamma(M)^{h}=0$.

\section{The structure theorem: statement and proof}

Definition 4.1 Given $f \in C^{2}(\Omega)$ and $F \in C^{1}\left(\Omega, \mathbb{R}^{2 m}\right)$, where $\Omega$ is an open subset of $\mathbb{R}^{2 m}$, define the sets

$$
A_{f, F}:=\{x \in \Omega \mid D f(x)=F(x)\}
$$

and

$$
A_{f, F}^{*}:=\left\{x \in A_{f, F} \mid \Gamma(D F(x))^{m} \neq 0\right\} .
$$

Remark 4.1 As we have already pointed out in Introduction, under the assumptions of Definition 4.1 , a point $x_{0} \in \Omega$ cannot be a $(2 m+1)$-density point of the set $A_{f, F}$ if $\Gamma\left(D F\left(x_{0}\right)\right) \neq 0$ (by [3, Theorem 2.1]). In particular one has the following property: There is no point $x_{0} \in A_{f, F}^{*}$ which is a $(2 m+1)$-density point of the $\operatorname{set} A_{f, F}^{*}$. In our main result, namely Theorem 4.1, we will prove that something very much stronger than this property actually holds for $A_{f, F}^{*}$. In particular, the set $A_{f, F}^{*}$ must be so thin that its Hausdorff dimension cannot exceed $m$.

We need the following lemma which will be proved by a simple argument combining Proposition 3.1 and the implicit function theorem.

Lemma 4.1 Let $\Phi \in C^{1}\left(\Omega, \mathbb{R}^{2 m}\right)$, where $\Omega$ is an open subset of $\mathbb{R}^{2 m}$. Then

$$
\left\{x \in \Omega \mid \Phi(x)=0, \Gamma(D \Phi(x))^{m} \neq 0\right\}
$$

is a relatively closed subset of $\Omega$ which is covered by a finite family $\left\{\Sigma_{\alpha} \mid \alpha \in I(2 m, m)\right\}$ of $m$-dimensional regularly imbedded $C^{1}$ submanifolds of $\mathbb{R}^{2 m}$. 
Proof For $\alpha \in I(2 m, m)$, define

$$
\Phi_{\alpha}:=\left(\Phi_{\alpha_{1}}, \ldots, \Phi_{\alpha_{m}}\right), \quad \Sigma_{\alpha}:=\left\{x \in \Omega \mid \Phi_{\alpha}(x)=0, \operatorname{rank} D \Phi_{\alpha}(x)=m\right\} .
$$

Observe that each $\Sigma_{\alpha}$ has to be a $m$-dimensional regularly imbedded $C^{1}$ submanifold of $\mathbb{R}^{2 m}$ by a standard application of the implicit function theorem, e.g., compare [6, Theorem 4.3.1] or [5, Ch. 1, Theorem 3.2]. The conclusion follows from the last identity in Proposition 3.1, with $M=D \Phi(x)$.

Now we are ready to prove our main result.

Theorem 4.1 (Structure theorem) Let $f \in C^{2}(\Omega)$ and $F \in C^{1}\left(\Omega, \mathbb{R}^{2 m}\right)$, where $\Omega$ is an open subset of $\mathbb{R}^{2 m}$. Then $A_{f, F}^{*}$ is a relatively closed subset of $\Omega$ which is covered by a finite family $\left\{\Sigma_{\alpha} \mid \alpha \in I(2 m, m)\right\}$ of m-dimensional regularly imbedded $C^{1}$ submanifolds of $\mathbb{R}^{2 m}$. In particular, the Hausdorff dimension of $A_{f, F}^{*}$ has to be less or equal to $m$.

Proof Consider

$$
\Phi:=D f-F \in C^{1}\left(\Omega, \mathbb{R}^{2 m}\right)
$$

Since

$$
D \Phi=D^{2} f-D F
$$

one has

$$
\Gamma\left(D \Phi(x)-(D \Phi(x))^{t}\right)=\Gamma\left((D F(x))^{t}-D F(x)\right)
$$

for all $x \in \Omega$. By recalling Remark 3.1, we get

$$
\Gamma(D \Phi(x))=-\Gamma(D F(x))
$$

for all $x \in \Omega$, hence

$$
A_{f, F}^{*}=\left\{x \in \Omega \mid \Phi(x)=0, \Gamma(D \Phi(x))^{m} \neq 0\right\} .
$$

The conclusion follows from Lemma 4.1.

From Theorem 4.1, we obtain the following corollary.

Corollary 4.1 Consider $F_{0}: \mathbb{R}^{2 m} \rightarrow \mathbb{R}^{2 m}$ defined as

$$
F_{0}\left(x_{1}, \ldots, x_{2 m}\right):=\left(2 x_{m+1}, \ldots, 2 x_{2 m},-2 x_{1}, \ldots,-2 x_{m}\right)
$$

and let $\Omega$ be an open subset of $\mathbb{R}^{2 m}$. Then, for all $f \in C^{2}(\Omega)$, the set $A_{f, F_{0}}$ is a relatively closed subset of $\Omega$ which is covered by a finite family $\left\{\Sigma_{\alpha} \mid \alpha \in I(2 m, m)\right\}$ of m-dimensional regularly imbedded $C^{1}$ submanifolds of $\mathbb{R}^{2 m}$. In particular, the Hausdorff dimension of $A_{f, F_{0}}$ has to be less or equal to $\mathrm{m}$.

Proof Observe that

$$
D F_{0}=\left(\begin{array}{cc}
0 & 2 I_{m} \\
-2 I_{m} & 0
\end{array}\right)
$$


where $I_{m}$ denotes the identity $m \times m$ matrix. Thus, for all $x \in \mathbb{R}^{2 m}$, we obtain

$$
\begin{aligned}
\Gamma\left(D F_{0}(x)\right) & =\sum_{i=1}^{2 m} D F_{0}(x) e_{i} \wedge e_{i}=\sum_{i=1}^{m}\left(-2 e_{m+i}\right) \wedge e_{i}+\sum_{i=m+1}^{2 m} 2 e_{i-m} \wedge e_{i} \\
& =4 \sum_{i=1}^{m} e_{i} \wedge e_{m+i}
\end{aligned}
$$

hence

$$
\begin{aligned}
\Gamma\left(D F_{0}(x)\right)^{m} & =4^{m} \sum_{i_{1}, \ldots, i_{m}=1}^{m}\left(e_{i_{1}} \wedge e_{m+i_{1}}\right) \wedge \cdots \wedge\left(e_{i_{m}} \wedge e_{m+i_{m}}\right) \\
& =4^{m} \sum_{\lambda \in S_{m}}\left(e_{\lambda(1)} \wedge e_{m+\lambda(1)}\right) \wedge \cdots \wedge\left(e_{\lambda(m)} \wedge e_{m+\lambda(m)}\right) \\
& =4^{m} \sum_{\lambda \in S_{m}}\left(e_{1} \wedge e_{m+1}\right) \wedge \cdots \wedge\left(e_{m} \wedge e_{2 m}\right) \\
& =4^{m} m !(-1)^{\frac{m(m-1)}{2}} e_{1} \wedge \cdots \wedge e_{2 m} .
\end{aligned}
$$

In particular one has $\Gamma\left(D F_{0}(x)\right)^{m} \neq 0$ for all $x \in \mathbb{R}^{2 m}$, so that $A_{f, F_{0}}=A_{f, F_{0}}^{*}$. The conclusion follows from Theorem 4.1.

Remark 4.2 Corollary 4.1 improves slightly the second statement in [1, Theorem 3.1], which yields immediately the following interesting fact: the characteristic set of a codimension 1 submanifold of class $C^{2}$ in the Heisenberg group $\mathbb{H}^{m}$ has Hausdorff dimension less or equal to $m$, compare [1, Theorem 1.2].

\section{References}

1. Balogh, Z.M.: Size of characteristic sets and functions with prescribed gradient. J. reine angew. Math. 564, 63-83 (2003)

2. Balogh, Z.M., Pintea, C., Rohner, H.: Size of tangencies to non-involutive distributions. Indiana Univ. Math. J. 60(6), 2061-2092 (2011)

3. Delladio, S.: Functions of class $C^{1}$ subject to a Legendre condition in an enhanced density set. Rev. Mat. Iberoam. 28(1), 127-140 (2012)

4. Franchi, B., Serapioni, R., Serra, F.: Cassano: on the structure of finite perimeter sets in step 2 Carnot groups. J. Geom. Anal. 13(3), 421-466 (2003)

5. Hirsch, M.W.: Differential Topology. Springer, Berlin (1976)

6. Krantz, S.G., Parks, H.R.: The Implicit Function Theorem. History, Theory and Applications. Modern Birkhäuser Classics. Birkhäuser, Basel (2013)

7. Magnani, V.: Characteristic points, rectifiability and perimeter measure on stratified groups. J. Eur. Math. Soc. (JEMS) 8(4), 585-609 (2006) 